The Astrophysical Journal, 664:377-383, 2007 July 20

(C) 2007. The American Astronomical Society. All rights reserved. Printed in U.S.A.

\title{
STAR FORMATION IN THE BOK GLOBULE CB54
}

\author{
DAVID R. CIARDI \\ Michelson Science Center/Caltech, M/S 100-22, Pasadena, CA 91125; ciardi@ipac.caltech.edu \\ AND \\ Cynthia Gómez Martín \\ Department of Astronomy, University of Florida, Gainesville, FL 32611; gomez@astro.ufl.edu \\ Received 2007 March 1; accepted 2007 April 2
}

\begin{abstract}
We present mid-infrared $(10.4,11.7$, and $18.3 \mu \mathrm{m})$ imaging intended to locate and characterize the suspected protostellar components within the Bok globule CB54. We detect and confirm the protostellar status for the nearinfrared source CB54YC1-II. The mid-infrared luminosity for CB54YC1-II was found to be $L_{\text {midir }} \approx 8 L_{\odot}$, and we estimate a central source mass of $M_{*} \approx 0.8 M_{\odot}$ (for a mass accretion rate of $\dot{M}=10^{-6} M_{\odot} \mathrm{yr}^{-1}$ ). CB54 harbors another near-infrared source (CB54YC1-I), which was not detected by our observations. The nondetection is consistent with CB54YC1-I being a highly extinguished embedded young A or B star or a background G or F giant. An alternative explanation for CB54YC1-I is that the source is an embedded protostar viewed at an extremely high inclination angle, and the near-infrared detections are not of the central protostar, but of light scattered by the accretion disk into our line of sight. In addition, we have discovered three new mid-infrared sources, which are spatially coincident with the previously known dense core in CB54. The source temperatures $(\sim 100 \mathrm{~K})$ and association of the mid-infrared sources with the dense core suggests that these mid-infrared objects may be embedded class 0 protostars.
\end{abstract}

Subject headings: infrared: ISM — infrared: stars — ISM: globules — ISM: individual (CB54, LBN 1042) — stars: formation - stars: pre-main-sequence

\section{INTRODUCTION}

Protostars are young stellar objects that are still in the process of accreting the bulk of their material. Class 0 sources have been proposed as the evolutionary precursors to the class I protostars (André et al. 1993; André \& Montmerle 1994). Work by Bontemps et al. (1996) and Saraceno et al. (1996) suggested a direct evolutionary sequence from the class 0 stage to the class I stage. However, Jayawardhana et al. (2001) have argued that class 0 protostars are located preferentially in higher density regions, and that class I young stellar objects are located preferentially in lower density regions and, thus, may be at comparable evolutionary states. To further complicate the distinction between class 0 and class I objects, some class I objects, which are viewed at high inclination, may appear "class 0-like" because of the high optical depth associated with viewing the disk (nearly) edge-on (Masunaga \& Inutsuka 2000).

An example of this confusion may be seen in the binary system L1448N(A, B), where one component of the system has the spectral energy distribution (SED) of a class 0 protostar, while the other has an SED of a class I protostar (Ciardi et al. 2003; O'Linger et al. 2006). This spread in apparent evolutionary status is also seen in larger clusters. In Perseus, Rebull et al. (2007) found that sources within clusters exhibited SEDs for a wide range in circumstellar environments, suggesting class 0 to class II protostars within the same aggregate. Either viewing geometry plays a significant role in our interpretation for each of the SEDs, the aggregates span a significant age spread, or if the sources are coeval, the disks/envelopes evolve faster than anticipated.

To help address these issues, it would be beneficial to study a set of young stellar objects, located within the same environment, but isolated and free from the influence of other active star formation. The Bok globule CB54, known to be a site of active star for- mation, may provide such an environment of isolated aggregate star formation.

CB54 (LBN 1042; Clemens \& Barvainis 1988) is a $\sim 100 M_{\odot}$ Bok globule associated with the Vela OB1 molecular cloud $(d \approx$ 1500 pc; Launhardt \& Henning 1997; Launhardt et al. 1997). Bok globules are small (10-100 $M_{\odot}$; Clemens et al. 1991), isolated molecular clouds, most of which have been identified via opaque patches in optical images (Clemens \& Barvainis 1988; Bourke et al. 1995). Globules have been found to be sites of star formation (e.g., Yun \& Clemens 1990, 1994a, 1994b; Alves \& Yun 1995; Moreira \& Yun 1995) of both single low-mass stars and multiple or binary stars (e.g., Yun 1996).

Active star formation in CB54 was first identified by the association of a dense core with the IRAS point source PSC 070201618 , which is located at the center of a collimated molecular outflow (Yun \& Clemens 1994b). Near-infrared imaging revealed that CB54 actually contains two bright near-infrared sources (CB54YC1-I, CB54YC1-II) and diffuse nebulosity of shocked $\mathrm{H}_{2}$ emission connecting the two sources (see Fig. 1 and Yun 1996; Khanzadyan 2003). The positions of CB54YC1-I and -II are offset from the position of the IRAS point source (see Fig. 1), although the IRAS beam size and PSC positional errors $\left(20^{\prime \prime} \times\right.$ $\left.4^{\prime \prime}\right)$ do make it difficult to associate any one source with IRAS 07020-1618.

Based on its near-infrared colors $(J-K=5.29 \mathrm{mag}, H-K=$ $2.58 \mathrm{mag}$ ), CB54YC1-II was classified as a class I young stellar object (Yun 1996). There is an MSX point source (G228.9946$04.6200)$, detected only in the A band $(8.3 \mu \mathrm{m})$, within a few arcseconds of CB54YC1-II (see Fig. 1). CB54YC1-I ( $J-K=$ $4.34 \mathrm{mag}, H-K=1.63 \mathrm{mag}$ ) was also classified as a class I protostar (Yun 1996), but despite its similar near-infrared brightness to CB54YC1-II, it was not detected by MSX. Unlike CB54YC1-II, the near-infrared colors of CB54YC1-I could 


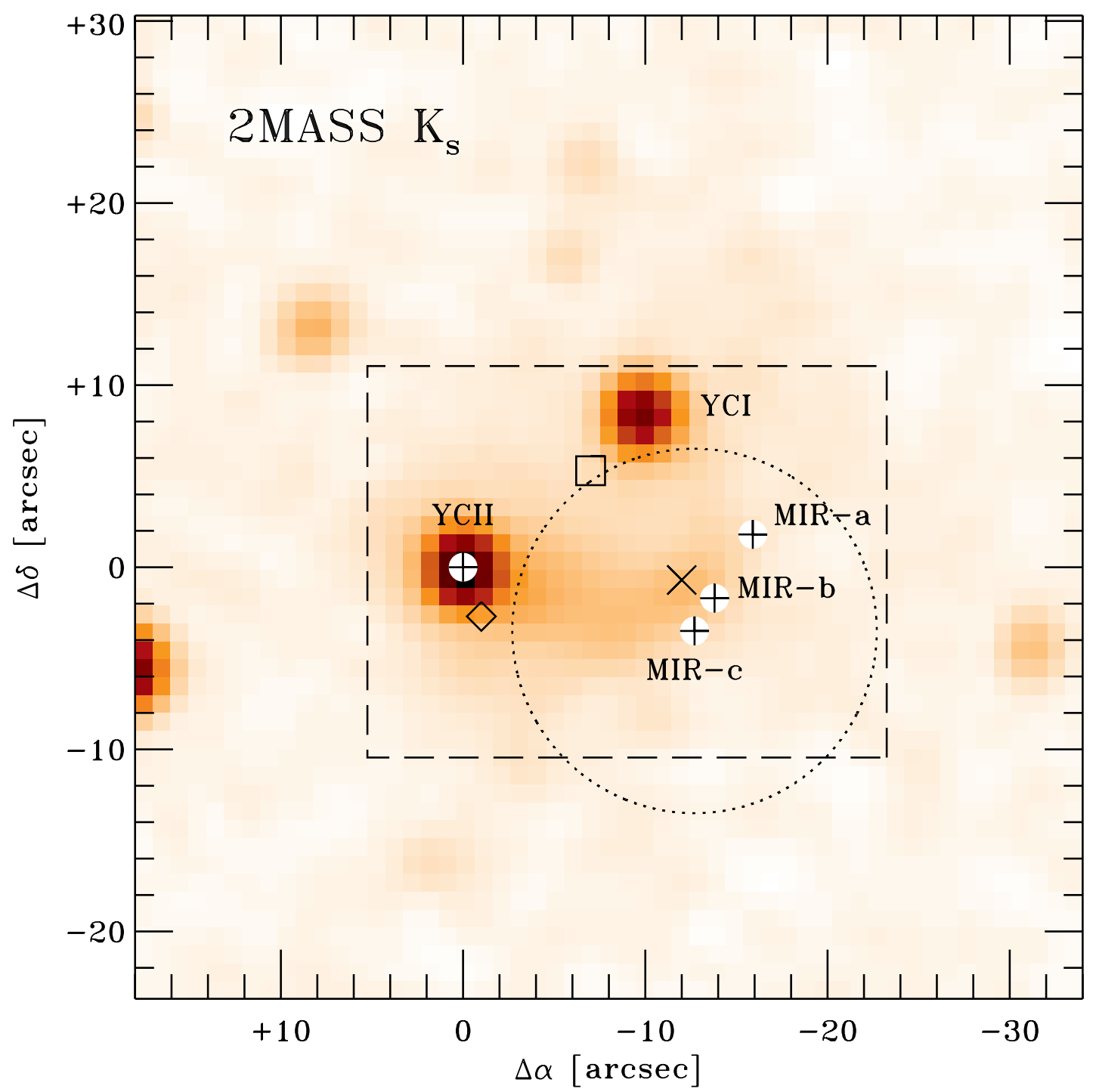

FIG. 1.-2MASS $K_{s}$ image of CB 54. The dashed line delineates the area imaged with T-ReCS. The near-infrared sources CB54YC1-I and CB54YC1-II are annotated, and the positions of the detected mid-infrared sources are marked with the filled white circles (see Fig. 2). The image $(0,0)$ position is centered on CB54YC1-II. The dotted circle is centered on the peak of $850 \mu \mathrm{m}$ core; the size of the circle represents the approximate size of the $850 \mu \mathrm{m}$ core (Henning et al. 2001). The cross marks the position of the IRAS source PSC $07020-1618$. The open diamond marks the position of the $8 \mu \mathrm{m}$ MSX source G228.9946-04.6200, and the open square marks the position for the $\mathrm{cm}$ source discovered with the VLA (Yun et al. 1996). The image pixel scale is $1^{\prime \prime}$ pixel $^{-1}$.

be explained with a highly extinguished $\left(A_{V} \sim 20 \mathrm{mag}\right)$ "bare" photosphere. VLA observations detected a $3.6 \mathrm{~cm}$ and $6 \mathrm{~cm}$ source within a few arcseconds of CB54YC1-I (see Fig. 1), possibly indicating a stellar wind or accretion shock (Yun et al. 1996; Moreira et al. 1997).

The position of the IRAS point source is spatially coincident with a dense core revealed in submillimeter, millimeter, and molecular line mapping; the position of which is offset from the positions of CB54YC1-I and CB54YC1-II (see Fig. 1; and, e.g., Wang et al. 1995; Zhou et al. 1996; Henning et al. 2001). Molecular line observations also indicated the presence of gravitational collapse in the core of CB54 (Wang et al. 1995; Afonso et al. 1998). Water maser emission, almost exclusively associated with class 0 protostars in regions of low-mass star formation (e.g., Furuya et al. 2001), was also discovered in CB54 (de GregorioMonsalvo et al. 2006; Gómez et al. 2006). All of this suggests that the star formation in CB54 may be more substantial than revealed by the near-infrared imaging alone.
We have observed the mid-infrared emission from the Bok globule CB54 at high spatial resolution $\left(\sim 0.5^{\prime \prime}\right)$ to clarify the evolutionary status of CB54YC1-I and CB54YC1-II and to search for additional protostars embedded in the globule core. Our work confirms the protostellar status of CB54YC1-II, but indicates that CB54YC1-I may be a more evolved young stellar object or a background giant star. In addition, we have discovered three new mid-infrared sources, which are spatially coincident with the dense core and may be class 0 protostars.

\section{OBSERVATIONS AND DATA REDUCTION}

Mid-infrared imaging observations of CB54 were made on 2004 February 01 (UT) using the Thermal Region Camera and Spectrograph (T-ReCS; Telesco et al. 1998) on the Gemini South $8 \mathrm{~m}$ telescope. T-ReCS utilizes a $320 \times 240$ pixel Si:As blocked impurity-band detector, with a spatial scale of $0.089^{\prime \prime} \mathrm{pixel}^{-1}$ and a field of view of $28.8^{\prime \prime} \times 21.6^{\prime \prime}$. The observations were centered on the $\mathrm{J} 2000$ coordinates $(\alpha, \delta)=\left(\alpha=07^{\mathrm{h}} 04^{\mathrm{m}} 21^{\mathrm{s}}\right.$, 
TABLE 1

Summary of Observations

\begin{tabular}{|c|c|c|c|c|c|}
\hline Filter & $\begin{array}{c}\lambda_{c} \\
(\mu \mathrm{m})\end{array}$ & $\begin{array}{c}\Delta \lambda \\
(\mu \mathrm{m})\end{array}$ & $\begin{array}{c}\text { Frame Time } \\
(\mathrm{ms})\end{array}$ & $\begin{array}{c}\text { On-Source } \\
\text { (s) }\end{array}$ & Air Mass \\
\hline N......................... & 10.36 & 5.27 & 25.8 & 304 & 1.11 \\
\hline Si-11.66 ........... & 11.7 & 1.13 & 25.8 & 304 & 1.84 \\
\hline Qa-18.30......... & 18.3 & 1.51 & 25.8 & 912 & $1.18-1.34$ \\
\hline
\end{tabular}

$\left.\delta=-16^{\circ} 23^{\prime} 19^{\prime \prime}\right)$. Imaging was obtained in three filters $(\mathrm{N}$, Si-11.7, and Qa-18.3). The on-sky alignment of the T-ReCS field of view was chosen to cover the entire near-infrared nebulosity, the two known near-infrared sources, and the peak of the submillimeter $(850 \mu \mathrm{m})$ core (see Fig. 1).

A standard off-chip $15^{\prime \prime}$ north-south chop-nod sequence was employed with total on-source integration times of $300 \mathrm{~s}$ per image. Three exposures in the Qa-18.3 filter were acquired for a total on-source integration time of $900 \mathrm{~s}$. Flux calibration was obtained from imaging of the standard star HD 32887 (see the Gemini Web page for a compilation of mid-infrared standard stars and flux densities). ${ }^{1}$ The weather quality was listed as the 50 th percentile, and the seeing at $11.7 \mu \mathrm{m}$ was $\approx 0.4^{\prime \prime}$. A summary of the filters, frame times, total integration time per filter, and associated air masses is given in Table 1.

The data were reduced with custom-written IDL routines for the T-ReCS data format. Four mid-infrared sources were detected by our observations, with no evidence of extended or diffuse midinfrared emission (see Fig. 2). Standard aperture photometry was performed using a $1^{\prime \prime}$ aperture radius. Detection limits were tested by inserting fake sources into the images and performing aperture photometry. A summary of the photometry (including estimated $1 \sigma$ upper limits), and relative positional offsets with respect to CB54YC1-II is given in Table 2.

\section{DISCUSSION}

The near-infrared source CB54YC1-II is the brightest midinfrared source and is detected in each of the mid-infrared filters. The other near-infrared source CB54YC1-I was not detected in any of the mid-infrared imaging. In addition, three new midinfrared sources have been detected (MIR-a, MIR-b, and MIR-c). MIR-a and MIR-b were detected in each of three mid-infrared filters, while MIR-c was detected only at $18.3 \mu \mathrm{m}$. In the following sections, we evaluate the properties of these sources and discuss the possible star formation history of the globule.

\subsection{CB54YC1-II}

CB54YC1-II, originally classified as a candidate class I protostar (Yun 1996), has a $2.2-10.3 \mu \mathrm{m}$ spectral index $[\alpha=$ $\left.-d \log \left(\nu F_{\nu}\right) / d \log (\nu)\right]$ of $\alpha=0.34 \pm 0.01$, which is consistent with the spectral index expected for a class I/flat-spectrum young stellar object. The SED of CB54YC1-II is shown in Figure 3. For comparison, the SED for a confirmed class I young stellar object (IRAS 04195+2251; Eisner, J. et al. 2005), with a similar spectral index $\alpha_{(2.2-10.6 \mu \mathrm{m})} \approx 0.3$, has been scaled to the SED of CB54YC1-II (see Fig. 3), exhibiting good agreement between the SEDs.

If the mid-infrared emission is primarily the result of gravitational infall (e.g., Ciardi et al. 2003), the mid-infrared luminosity provides a means of estimating the central protostellar mass. Integrating the SED from 1-20 $\mu \mathrm{m}$, we estimate the mid-infrared

\footnotetext{
1 See http://www.gemini.edu/sciops/instruments/mir/MIRPhotStandards.html.
}

luminosity to be $L_{\text {midir }} \approx 8 \pm 2 L_{\odot}$, for an assumed distance of $1500 \mathrm{pc}$. We estimate a central source mass from the relation $L=\left(G M M_{*}\right) / R_{*}$, where $\dot{M}$ is the mass infall rate, $R_{*}$ is the source size, and $M_{*}$ is the source mass (Shu et al. 1987). Using a standard $R_{*}=3 R_{\odot}$ protostellar radius (Stahler et al. 1980) and typical mass accretion rates of $\dot{M}=10^{-5}-10^{-6} M_{\odot} \mathrm{yr}^{-1}$ (Kenyon et al. 1993), we estimate the central protostellar mass for CB54YC1-II to be $M_{*}=0.08-0.8 M_{\odot}$.

A class II pre-main-sequence star located behind a wall of extinction could also explain the observed SED for CB54YC1-II. In Figure 3, a median SED for T Tauri stars (TTS; D'Alessio et al. $1999)$ has been scaled and convolved with an extinction model ( $R=3.1$ assumed, Mathis 1990). In order to match both the midinfrared flux densities and the slope of the near-infrared, the TTS SED must be extinguished by $A_{V} \approx 25 \mathrm{mag}$, and indeed, the position of CB54YC1-II in a $J H K$ color-color $(J-H=2.71 \mathrm{mag}$, $H-K=2.58 \mathrm{mag}$ ) diagram is consistent with a heavily extinguished TTS (see Fig. 4 in Haisch et al. 2000).

The average volume density of the CB54 envelope (i.e., not including the central condensation that is offset from the near-infrared sources), as derived from submillimeter (450 and $850 \mu \mathrm{m}$ ) imaging, is $\left\langle n_{\mathrm{H}}\right\rangle \approx 5 \times 10^{4} \mathrm{~cm}^{-3}$ (Henning et al. 2001). At $1500 \mathrm{pc}$, the projected linear radius of the CB54 envelope is $r \approx 22,500 \mathrm{AU}\left(\approx 15^{\prime \prime}\right)$. If we assume the globule is spherical, we can derive a peak column density of $N_{\mathrm{H}} \approx 4 \times 10^{22} \mathrm{~cm}^{-2}$, which corresponds to a visual extinction of $A_{V} \approx 20 \mathrm{mag}$. The extinction estimation does not take into account specific structure within the cloud including any dense envelope that may immediately surround the source, but does indicate the above derived extinction levels for CB54YC1-II are possible. Without a more complete SED or spectroscopy, especially at $3-8 \mu \mathrm{m}$, it is difficult to distinguish between the class I and class II models for CB54YC1-II

\section{2. $C B 54 Y C 1-I$}

CB54YC1-I was not detected in any of the three mid-infrared images, calling into question the original class I protostellar classification. Scaling the SED for the class I protostar (IRAS 04295+ 2251) to the $J H K$ SED of CB54YC1-I, the predicted mid-infrared flux densities for CB54YC1-I are $F_{\nu} \sim 80-100 \mathrm{mJy}$ at $11.7 \mu \mathrm{m}$ and $F_{\nu} \sim 150 \mathrm{mJy}$ at $18.3 \mu \mathrm{m}$. The predicted emission is $\sim 10 \sigma$ above the detection limits (see Fig. 4).

The $J H K$ slope for CB54YC1-I is too steep to match the nearinfrared SED for a class II (TTS) pre-main-sequence star. However, if the TTS SED is modified with a screen of foreground extinction, the near-infrared SED for CB54YC1-I can be reproduced with a class II pre-main-sequence model. In Figure 4, as was done for CB54YC1-II, the median TTS SED (D'Alessio et al. 1999) has been scaled and convolved with an extinction model and fitted to the $J H K$ data for CB54YC1-I. For a best-fit extinction of $A_{V}=15-17 \mathrm{mag}$, the TTS SED can reproduce the near-infrared data. However, the model predicts mid-infrared densities $\left(F_{\nu} \sim 20-40 \mathrm{mJy}\right.$ at $11.7 \mu \mathrm{m}$ and $F_{\nu} \sim 50-70 \mathrm{mJy}$ at $18.3 \mu \mathrm{m}), \sim 7 \sigma$ above the detection limits.

It is possible that CB54YC-I is a more evolved star embedded in the globule or simply a star background to the globule. To explore these possibilities, we have fitted the $J H K$ photometry with a blackbody function modified by a line-of-sight extinction curve: $S_{\nu}=\Omega B_{\nu}(T) \exp \left(-A_{\nu} / 1.086\right)$, where $B_{\nu}(T)$ is the Planck function, $A_{\nu}$ is the frequency-dependent extinction, and $\Omega$ is the solid angle. At each extinction value in the range from $A_{V}=0-30 \mathrm{mag}\left(\Delta A_{V}=0.1 \mathrm{mag}\right)$, a range of temperatures $(T=$ $500-50,000 \mathrm{~K}$ in steps of $100 \mathrm{~K}$ ) were tested. 

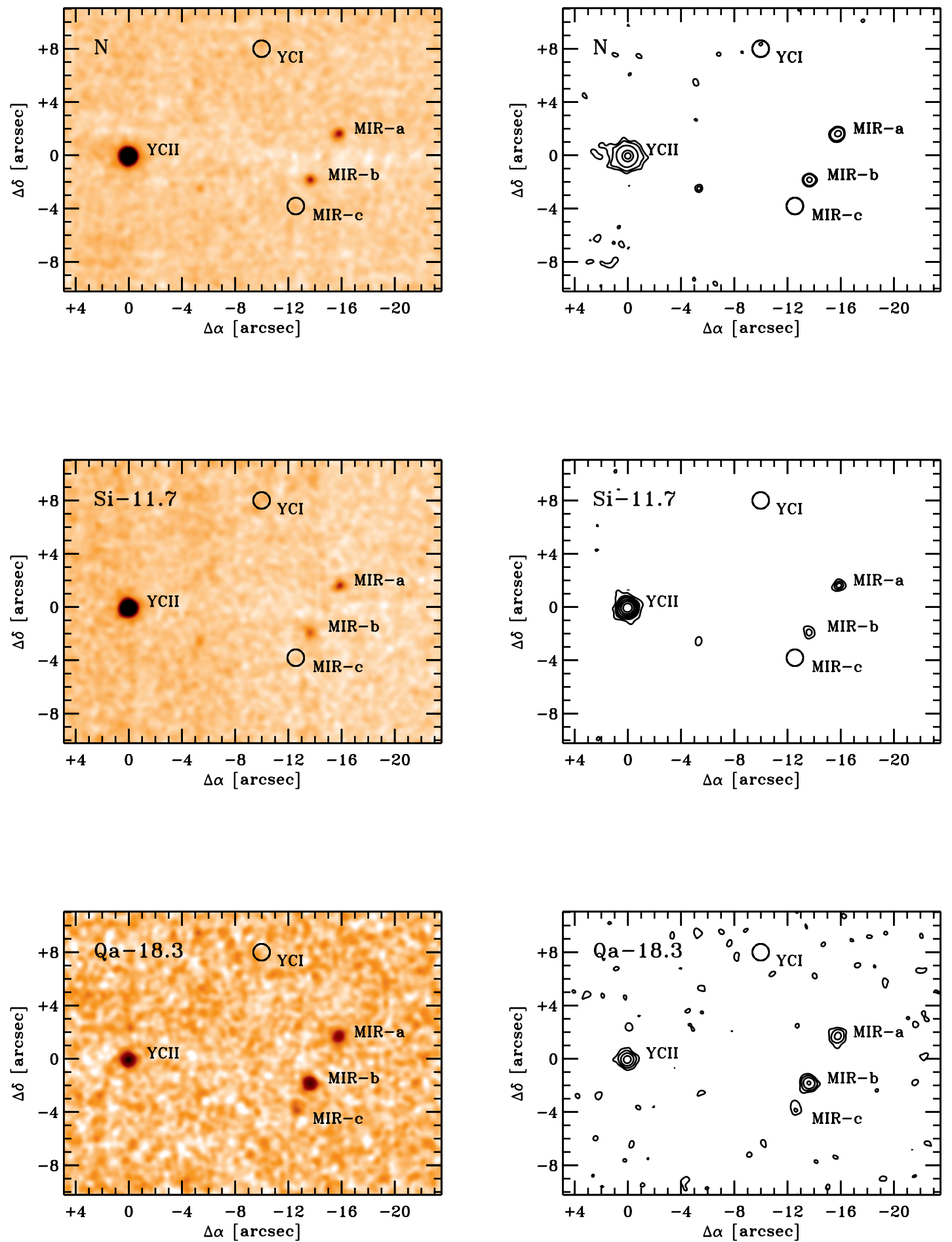

FIG. 2.-T-ReCS N-band, 11.7 and $18.3 \mu \mathrm{m}$ images and contour plots of CB54. The $(0,0)$ point of each image is centered on CB54YC1-II. The images have been stretched by an inverse hyperbolic sine to enhance the contrast. The detected mid-infrared sources are annotated. The position of CB54YC1-I (not detected by the mid-infrared observations) is marked in each image, and the position of MIR-c (detected only at $18.3 \mu \mathrm{m}$ ) is marked in the N-band and $11.7 \mu \mathrm{m}$ images. The N-band

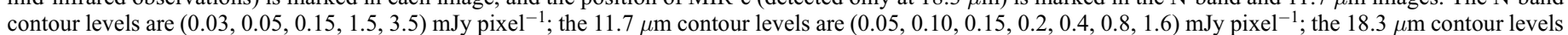
are $(0.4,0.8,1.6,3.2) \mathrm{mJy}_{\mathrm{pixel}}{ }^{-1}$. The pixel scale for each image is $0.089^{\prime \prime} \mathrm{pixel}^{-1}$.

For a given extinction value, there is a unique blackbody temperature for which the $\chi^{2}$ is a minimum, but there is no global minimum representing a best fit to the $J H K$ data. In Figure 5, the resulting reduced $\chi^{2}$ and temperatures for each of the trial extinctions are plotted. The fitting uncertainty in the temperature for a given extinction value is approximately $10 \%$. The reduced $\chi^{2}$ curve is relatively flat between $0 \leq A_{V} \leq 26 \mathrm{mag}$. Beyond $A_{V}=26 \mathrm{mag}$, the reduced $\chi^{2}$ climbs above $\chi_{\nu}^{2} \approx 1$ and begins to diverge rapidly. The best-fit temperature at this boundary is $T \approx 30,000 \mathrm{~K}$. Because of the rapid change in the $\chi^{2}$ beyond this point, we regard this as the upper bound for the extinction and source temperature of CB54YC1-I.

The lower bound to the extinction and temperature is constrained only by the detection limits of the mid-infrared observations. The combination of temperature and extinction must be such that CB54YC1-I is not detected in all three mid-infrared 


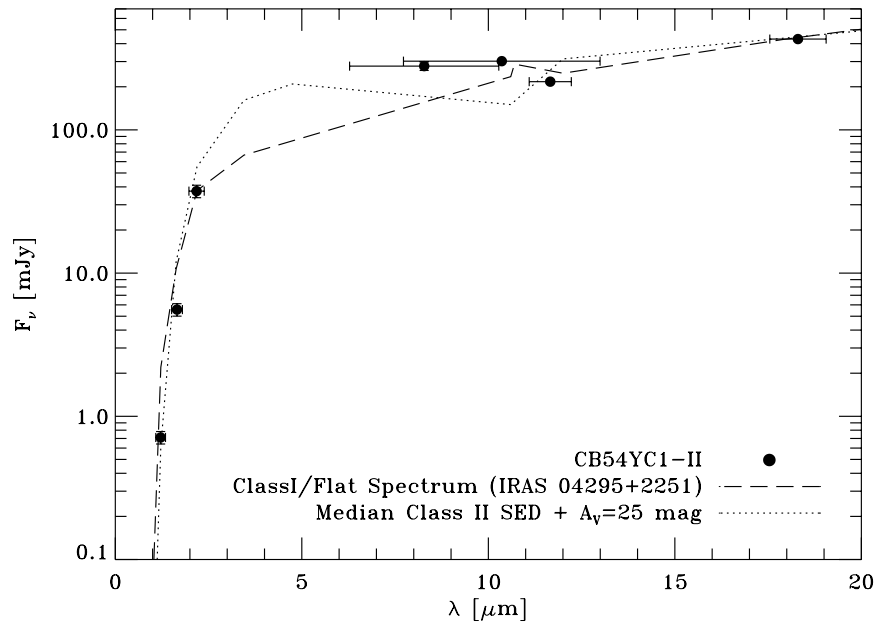

FIG. 3.-SED of CB54YC1-II ( filled circles). The horizontal error bars represent the bandwidth of the filters. The dashed line represents the SED for the class I protostar IRAS $04295+2251$. The dotted line represents a median TTS SED convolved with $A_{V}=25$ mag of extinction.

filters (N, Si-11.7, and Qa-18.3). For example, at zero extinction ( $A_{V}=0 \mathrm{mag}$ ), the best-fit temperature is $T \approx 1100 \mathrm{~K}$, and the reduced $\chi^{2}$ is $\chi_{\nu}^{2}<1$, but the predicted mid-infrared flux densities violate the nondetections at both $\mathrm{N}$ and $11.7 \mu \mathrm{m}$ (see Fig. 4).

The blackbody plus extinction models only predict midinfrared flux densities below the detection limits in all three midinfrared filters if $A_{V} \gtrsim 23 \mathrm{mag}$. As an example, the predicted $N$-band flux densities (the most sensitive of the three observations) are plotted as a function of visual extinction in Figure 5 (bottom), showing that the predicted flux density drops below the detection limit at $A_{V} \gtrsim 23 \mathrm{mag}$. We, therefore, regard $A_{V} \approx$ $23 \mathrm{mag}$ and the corresponding temperature $(T \approx 6000 \mathrm{~K})$ as the lower bound for CB54YC1-I. The lower $\left(A_{V}=23 \mathrm{mag}, T=\right.$ $6000 \mathrm{~K})$ and upper $\left(A_{V}=26 \mathrm{mag}, T=30,000 \mathrm{~K}\right)$ bounds for the blackbody model fits to CB54YC1-I are shown in Figure 4.

If the extinction is $A_{V}=23 \mathrm{mag}$, the temperature $(T \approx$ $6000 \mathrm{~K}$ ) suggests that the CB54YC1-I could be an early-G or late-F star. A main-sequence dwarf of this spectral type has an absolute $K$ magnitude of $M_{K} \approx 2.7 \mathrm{mag}$. With a measured $K$ magnitude of $K=11.76 \mathrm{mag}$ (Yun 1996), the implied distance is only $\sim 250 \mathrm{pc}$, much too close to be extinguished by CB54. If, however, CB54YC1-I is a G or F giant star, the star would be approximately $4.0 \mathrm{mag}$ brighter and located at a distance of $\approx 1500 \mathrm{pc}$, sufficiently distant to place CB54YC1-I behind the globule.

At the upper limit to the model fitting, $\left(A_{V} \approx 26 \mathrm{mag}, T \approx\right.$ $30,000 \mathrm{~K})$, the temperature corresponds to a B0 star. With an

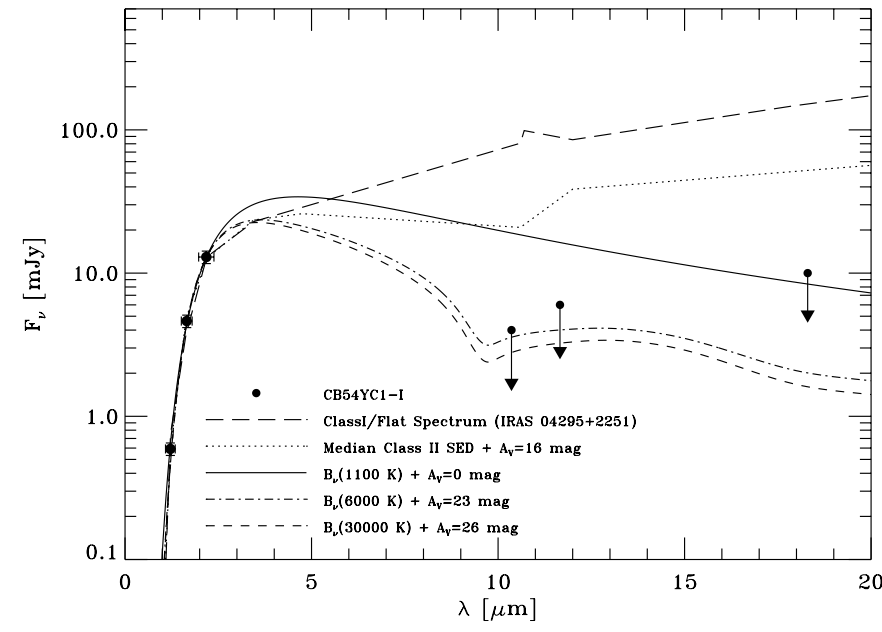

FIG. 4.-SED of CB54YC1-I ( filled circles). Upper limits for the midinfrared observations are represented by the down arrows. The long-dashed line represents the SED for the class I protostar IRAS $04295+2251$. The dotted line represents a median TTS SED convolved with $A_{V}=16$ mag of extinction. The solid line is a $1100 \mathrm{~K}$ blackbody; the dash-dotted line is a $6000 \mathrm{~K}$ blackbody convolved with $A_{V}=23 \mathrm{mag}$ of extinction, and the short-dashed line is a $30,000 \mathrm{~K}$ blackbody convolved with $A_{V}=26 \mathrm{mag}$ of extinction.

estimated absolute magnitude of $M_{K}=-3$, CB54YC1-I would be at a distance of $2500 \mathrm{pc}$, which is far enough to be behind the globule. The rarity and short lifespan (10 Myr) of B0 stars and the required chance alignment with the globule seem to make this a remote possibility.

If, instead, the extinction is near the middle of the extinction range $\left(A_{V}=24-25 \mathrm{mag}\right)$, the best-fit temperature $(T \approx 10,000$ $15,000 \mathrm{~K}$ ) implies that CB54YC1-I may be a young A or B star. With an absolute $K$ magnitude of $M_{K} \approx-1.5-0$, this yields a distance of 1200-1500 pc, placing CB54YC1-I at a distance consistent with being an embedded young star.

An alternative explanation for CB54YC1-I is that the source is an embedded protostar viewed at an extremely high inclination angle, and the near-infrared detections are not of the central protostar, but of light scattered by the accretion disk into our line of sight. Unfortunately, near-infrared photometry alone cannot distinguish these models.

\subsection{Mid-Infrared Sources}

Three new sources have been detected by our mid-infrared observations. These sources have no near-infrared counterparts. The mid-infrared sources lie just beyond the edge of the shocked $\mathrm{H}_{2}$ emission and do not correspond to any of the knots or condensations visible in the near-infrared diffuse emission (see Fig. 1

TABLE 2

Source Positions and Flux Densities

\begin{tabular}{|c|c|c|c|c|c|c|c|c|c|}
\hline Source & $(\Delta \alpha, \Delta \delta)^{\mathrm{a}}$ & $\begin{array}{c}J \\
1.22 \mu \mathrm{m} \\
(\mathrm{mJy})\end{array}$ & $\begin{array}{c}H \\
1.65 \mu \mathrm{m} \\
(\mathrm{mJy})\end{array}$ & $\begin{array}{c}K \\
2.18 \mu \mathrm{m} \\
(\mathrm{mJy})\end{array}$ & $\begin{array}{c}\text { MSX-A } \\
8.28 \mu \mathrm{m} \\
(\mathrm{mJy})\end{array}$ & $\begin{array}{c}\mathrm{N} \\
10.36 \mu \mathrm{m} \\
(\mathrm{mJy})\end{array}$ & $\begin{array}{c}\mathrm{Si}-11.7 \\
11.66 \mu \mathrm{m} \\
(\mathrm{mJy})\end{array}$ & $\begin{array}{c}\text { Qa-18.3 } \\
18.3 \mu \mathrm{m} \\
(\mathrm{mJy})\end{array}$ & References \\
\hline YCII ................... & $(0.0,0.0)$ & $0.71 \pm 0.07$ & $5.56 \pm 0.56$ & $37.3 \pm 3.7$ & $278 \pm 19$ & $302 \pm 5$ & $217 \pm 6$ & $431 \pm 11$ & 1,2 \\
\hline 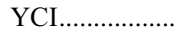 & $(-10.0,+8.3)$ & $0.59 \pm 0.06$ & $4.63 \pm 0.46$ & $12.9 \pm 1.3$ & $\ldots$ & $<4$ & $<6$ & $<10$ & 1,2 \\
\hline MIR-a .............. & $(-15.8,+1.7)$ & $\ldots$ & $\ldots$ & $\ldots$ & $\ldots$ & $16 \pm 4$ & $14 \pm 6$ & $219 \pm 11$ & 2 \\
\hline MIR-b.............. & $(-13.6,-1.8)$ & $\ldots$ & $\ldots$ & $\ldots$ & $\ldots$ & $12 \pm 4$ & $14 \pm 6$ & $322 \pm 11$ & 2 \\
\hline MIR-c ............... & $(-12.6,-3.8)$ & $\ldots$ & $\ldots$ & $\ldots$ & $\ldots$ & $<4$ & $<6$ & $70 \pm 12$ & 2 \\
\hline
\end{tabular}

${ }^{\mathrm{a}}$ Positional offsets in arcseconds from the source CB54YC1-II $\left[\alpha=07^{\mathrm{h}} 04^{\mathrm{m}} 21.7^{\mathrm{s}}, \delta=-16^{\circ} 23^{\prime} 19^{\prime \prime}(\mathrm{J} 2000)\right]$.

REFERENCES.-(1) Yun 1996; (2) This Work. 

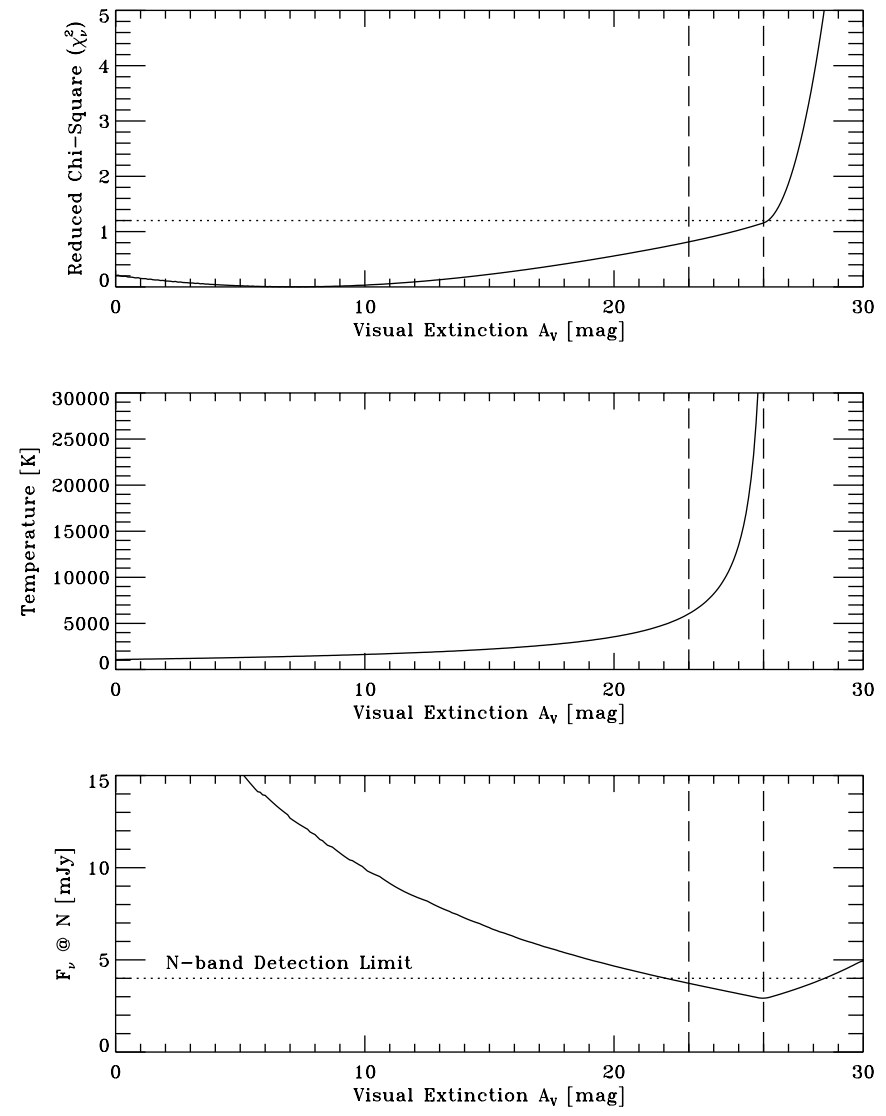

FIG. 5.- Reduced $\chi^{2}$ (top), best-fit temperatures (middle), and predicted $\mathrm{N}$-band flux densities (bottom) are plotted as a function of visual extinction for the blackbody+extinction models fitted to the JHK flux densities of CB54YC1-I. The horizontal dashed line in the $\chi^{2}$ plot marks the sharp knee in the $\chi^{2}$ curve at $A_{V}=26 \mathrm{mag}\left(\chi_{\nu}^{2}=1.2\right)$. The horizontal dashed line in the flux density plot marks the $1 \sigma$ detection limit ( $4 \mathrm{mJy}$ ) of the $\mathrm{N}$-band observations. The vertical dashed lines delineate the extinction range of $23 \leq A_{V} \leq 26 \mathrm{mag}$ (see text for details).

and Yun 1996; Khanzadyan 2003). The mid-infrared sources, however, are located within the boundaries of the dense core in CB54 and clustered near the position of the IRAS point source (see Fig. 1). The two brightest sources (MIR-a, MIR-b) were detected in all three filters, but MIR-c was detected only at $18.3 \mu \mathrm{m}$. A summary of the photometry is given in Table 2, and the SEDs for these sources are presented in Figure 6.

To characterize and understand the relative temperatures of the mid-infrared sources, a single temperature blackbody was fit to both MIR-a and MIR-b. The blackbody fits did not include the broadband $\mathrm{N}(10.3 \mu \mathrm{m})$ flux densities, which are potentially contaminated with an unknown amount of amorphous silicate, and were fit to the narrowband 11.7 and $18.3 \mu \mathrm{m}$ flux densities. The best-fit blackbody (Fig. 6) temperatures for the two sources are quite similar $\left(T_{A}=110 \pm 10 \mathrm{~K}\right.$ and $\left.T_{B}=100 \pm 10 \mathrm{~K}\right)$ and are near what is expected for the bolometric temperatures of class 0 protostars (André et al. 2000). If the $\mathrm{N}$-band photometry is included in the fits, the resulting temperatures increase by $10-20 \mathrm{~K}$.

Blackbody fits to the submillimeter and millimeter emission from the dense core yields a much colder envelope temperature of $25 \mathrm{~K}$ (Launhardt et al. 1997). The summed flux densities predicted by the mid-infrared blackbody fits is not sufficient to explain the $100 \mu \mathrm{m}$ flux density for IRAS PSC $07020-1618\left(F_{\nu} \approx\right.$ $100 \mathrm{Jy}$ ), indicating that these class 0 protostars are harbored within the cold, dense envelope.

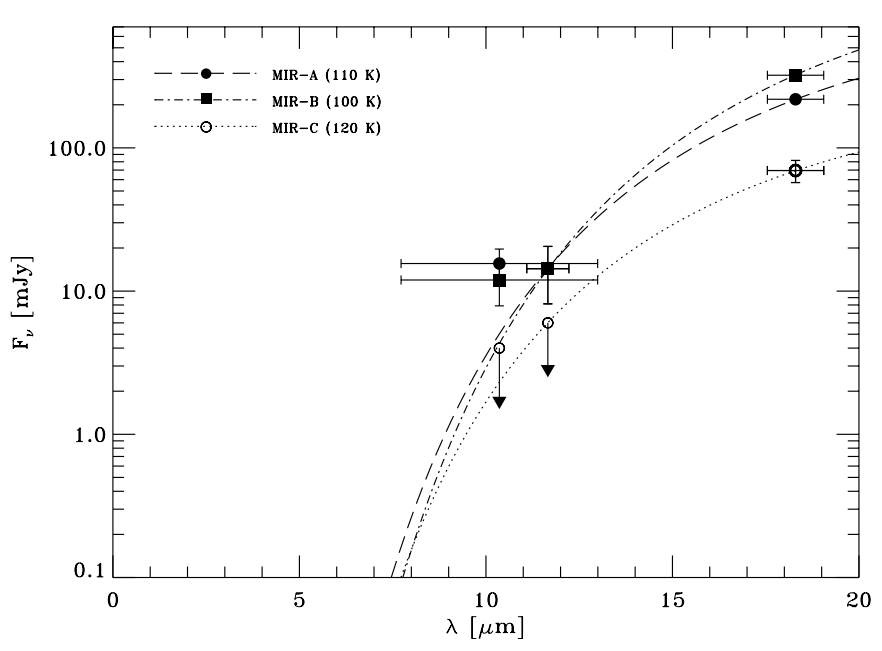

FIG. 6.-SEDs of the mid-infrared sources MIR-a (solid squares), MIR-b (solid circles), and MIR-c (open circles). The 110 and $100 \mathrm{~K}$ best-fit blackbody curves are shown for MIR-a (dashed) and MIR-b (dot-dashed). For MIR-c, the dotted line represents a $120 \mathrm{~K}$ blackbody, which is fit to the $18.3 \mu \mathrm{m}$ flux density and the $11.7 \mu \mathrm{m}$ upper limit. The horizontal error bars represent the bandwidth of the filters.

If we assume that the mid-infrared emission is optically thin and little emission is contributed from the cold envelope, we can estimate the protostellar core mass associated with mid-infrared emission via

$$
M_{d}=\frac{(16 / 3) \pi a \rho D^{2}}{Q_{\nu} B_{\nu}\left(T_{d}\right)} F_{\nu}
$$

where $F_{\nu}$ is the observed flux density at frequency $\nu, Q_{\nu}$ is the grain emissivity at frequency $\nu, a$ is the grain radius, $\rho$ is the grain mass density, $D$ is the distance to CB54, and $B_{\nu}$ is the Planck function at dust temperature $T_{d}$. Assuming $a=$ $0.5 \mu \mathrm{m}, \rho=1 \mathrm{~g} \mathrm{~cm}^{-3}, Q_{\nu}=0.1(\lambda / \mu \mathrm{m})^{-\alpha}$, and $\alpha=0.45$ (e.g., Muthumariappan et al. 2006), we estimate dust masses for MIR-a and MIR-b of $M_{a} \approx 0.014 M_{\odot}$ and $M_{b} \approx 0.043 M_{\odot}$, respectively. For an average gas-to-dust mass ratio of 100 , the central midinfrared cores have masses of $M_{a} \approx 1.4 M_{\odot}$ and $M_{b} \approx 4.3 M_{\odot}$.

MIR-c was detected only at $18.3 \mu \mathrm{m}$, but if we use the $11.7 \mu \mathrm{m}$ upper limit to restrict the blackbody fitting, we find an upper limit to the temperature of $120 \mathrm{~K}$. (see Fig. 6). Coupled with the $18.3 \mu \mathrm{m}$ flux density, we estimate a limit to the total mass (gas+dust) of $M_{c} \gtrsim 0.2 M_{\odot}$.

\section{CONCLUSIONS}

We have obtained high angular resolution $10-18 \mu \mathrm{m}$ imaging of CB54, a Bok globule known to harbor a dense core and two near-infrared sources previously classified as class I young stellar objects. We have detected only one (CB54YC1-II) of the two near-infrared sources, confirming its protostellar evolutionary status. Based on the mid-infrared luminosity, we estimate that the central protostellar mass for CB54YC1-II is $M_{*}=0.08-0.8 M_{\odot}$, depending on the mass transfer rate. The SED is also consistent with a more evolved T Tauri star behind a screen of extinction. Without a more complete SED, it is not possible to distinguish between these models.

The other near-infrared source (CB54YC1-I) should have been detected if it were a class I protostar similar to that of CB54YC1-II. We find that the near-infrared SED is consistent with the SED for a more evolved star extinguished by the globule itself. CB54YC1-I 
may be a background $\mathrm{F}$ or $\mathrm{G}$ giant or may be an embedded young A or B star. An alternative explanation for CB54YC1-I is that the source is an embedded protostar viewed at an extremely high inclination angle, and the near-infrared detections are not of the central protostar, but of light scattered by the accretion disk into our line of sight. High spatial resolution near-infrared polarimetry and/or mid-infrared spectroscopy could be used to ascertain the status CB54YC1-I. If CB54YC1-I is an embedded, young A or B star, its mass may be on the order of $\sim 2-5 M_{\odot}$.

In addition, we have discovered three new mid-infrared sources (MIR-a, MIR-b, and MIR-c), which are spatially coincident with both the position of the associated IRAS point source and the center of the dense core in CB54. These sources are characterized with a $100 \mathrm{~K}$ blackbody, consistent the expected bolometric temperature of a class 0 protostar. Based on the mid-infrared emission, we have estimated the masses for these sources to be $\sim 4$, $\sim 1.5$, and $\sim 0.2 M_{\odot}$.

If CB54YC1-I is indeed an embedded A or B star, it is interesting to speculate that CB54YC1-I may have formed first and induced star formation further in the cloud, through the interaction of its outflow/winds with remainder of the globule. The total mass estimated for the sources within CB54 is about $10-15 M_{\odot}$ or about $10 \%-15 \%$ of the total cloud mass. Such a sequential process of star formation occurring in the Bok globule CB54 may be similar to what is observed in other globules (Huard et al. 2000; Codella et al. 2006). Spectroscopy and a more complete SED in the mid-infrared and far-infrared is needed to disentangle the possible spectral types and evolutionary states for the sources in CB54.

These observations were carried out during payback time to $\mathrm{C}$. Telesco for development of T-ReCS. The authors would like to thank Charlie Telesco, Chris Packham, and Margaret Moerchen for collecting these data. Portions of this work were supported by the California Institute of Technology under contract with the National Aeronautics and Space Administration. C. G. M. acknowledges support from a University of Florida Graduate Minority Fellowship, a SEAGEP Fellowship, and NSF grants AST97-3367 and AST 02-02976. C. G. M. would like to thank Eric McKenzie and Ana Matkovic for comments and suggestions. Based on observations obtained at the Gemini Observatory, which is operated by the Association of Universities for Research in Astronomy, Inc., under a cooperative agreement with the NSF on behalf of the Gemini partnership: the National Science Foundation (United States), the Particle Physics and Astronomy Research Council (United Kingdom), the National Research Council (Canada), CONICYT (Chile), the Australian Research Council (Australia), CNPq (Brazil), and CONICET (Argentina). This research has made use of the NASA/IPAC Infrared Science Archive, which is operated by the Jet Propulsion Laboratory, California Institute of Technology, under contract with the National Aeronautics and Space Administration.
Afonso, J. M., Yun, J. L., \& Clemens, D. P. 1998, AJ, 115, 1111

Alves, J. F., \& Yun, J. L. 1995, ApJ, 438, L107

André, P., \& Montmerle, Th. 1994, ApJ, 420, 837

André, P., Ward-Thompson, D., \& Barsony, M. 1993, ApJ, 406, 122 2000, in Protostars and Planets IV, ed. V. Mannings, A. P. Boss, \&

S. S. Russell (Tucson: Univ. Arizona Press), 59

Bontemps, S., André, P., Terebey, S., \& Cabrit, S. 1996, A\&A, 311, 858

Bourke, T. L., Hyland, A. R., \& Robinson, G. 1995, MNRAS, 276, 1052

Ciardi, D. R., Telesco, C. M., Williams, J. P., Fisher, R. S., Packham, C., Piña, R., \& Radomski, J. 2003, ApJ, 585, 392

Clemens, D. P., \& Barvainis, R. 1988, ApJS, 68, 257

Clemens, D. P., Yun, J., \& Heyer, M. 1991, ApJS, 75, 877

Codella, C., Brand, J., Massi, F., Wouterloot, J. G. A., \& Davis, G. R. 2006, A\&A, 457, 891

D’Alessio, P., Calvet, N., Hartmann, L., Lizano, S., \& Cantó, J. 1999, ApJ, 527, 893

de Gregorio-Monsalvo, I., Gómez, J. F., Suárez, O., Kuiper, T. B. H., Anglada, G., Patel, N. A., \& Torrelles, J. M. 2006, AJ, 132, 2584

Eisner, J., et al. 2005, ApJ, 635, 396

Furuya, R. S., Kitamura, Y., Wootten, H. A., Claussen, M. J., \& Kawabe, R. 2001, ApJ, 559, L143

Gómez, J. F., de Gregorio-Monsalvo, I., Suárez, O., \& Kuiper, T. B. H. 2006, AJ, 132, 1322

Haisch, K. E., Jr., Lada, E. A., \& Lada, C. J. 2000, AJ, 120, 1396

Henning, Th., Wolf, S., Launhardt, R., \& Waters, R. 2001, ApJ, 561, 871

Huard, T. L., Weintraub, D. A., \& Sandell, G. 2000, A\&A, 362, 635

Jayawardhana, R., Hartmann, L., \& Calvet, N. 2001, ApJ, 548, 310

\section{REFERENCES}

Kenyon, S. J., Calvet, N., \& Hartmann, L. 1993, ApJ, 414, 676

Khanzadyan, T. 2003, Ph.D. thesis, Queen's Univ., Belfast

Launhardt, R., \& Henning, Th. 1997, A\&A, 326, 329

Launhardt, R., Ward-Thompson, D., \& Henning, Th. 1997, MNRAS, 288, L45

Masunaga, H., \& Inutsuka, S. 2000, ApJ, 531, 350

Mathis, J. S. 1990, ARA\&A, 28, 37

Moreira, M. C., \& Yun, J. L. 1995, ApJ, 454, 850

Moreira, M. C., Yun, J. L., Vázquez, R., \& Torrelles, J. M. 1997, AJ, 113, 1371

Muthumariappan, C., Kwok, S., \& Volk, K. 2006, ApJ, 640, 353

O’Linger, J. C., Cole, D. M., Ressler, M. E., \& Wolf-Chase, G. 2006, AJ, 131, 2601

Rebull, L., et al. 2007, ApJS, in press (astro-ph/0701711)

Saraceno, P., André, P., Ceccarelli, C., Griffin, M., \& Molinari, S. 1996, A\&A, 309,827

Shu, F. H., Adams, F. C., \& Lizano, S. 1987, ARA\&A, 25, 23

Stahler, S., Shu, F. H., \& Taam, R. E. 1980, ApJ, 242, 226

Telesco, C. M., Piña, R. K., Hanna, K. T., Julian, J. A., Hon, D. B., \& Kisko, T. M. 1998, Proc. SPIE, 3354, 534

Wang, Y., Evans, N. J., II, Zhou, S., \& Clemens, D. P. 1995, ApJ, 454, 217

Yun, J. L. 1996, AJ, 111, 930

Yun, J. L., \& Clemens, D. P. 1990, ApJ, 365, L73

1994a, AJ, 108, 612

1994b, ApJS, 92, 145

Yun, J. L., Moreira, M. C., Torrelles, J. M., Afonso, J. M., \& Santos, N. C. 1996, AJ, 111, 841

Zhou, S., Evans, N. J., II, \& Wang, Y. 1996, ApJ, 466, 296 